\title{
Hemiparkinsonism-Somatic Hemiatrophy Syndrome
}

\author{
Mary Jenkins, Dan Mendonca, Andrew Parrent, Mandar S. Jog
}

\begin{abstract}
Purpose: This paper highlights the hemiparkinsonism-hemiatrophy (HPHA) syndrome as a unique presentation of the parkinsonian state. Clinically relevant diagnostic and treatment aspects are reviewed. Method: We report a case of HPHA, in a 21-year-old, otherwise healthy, woman. Clinical and radiographic features of our case are presented. We also review the current literature on the clinical, radiological and pathophysiological mechanisms of HPHA. Results: In our case, despite the lack of benefit from anticholinergics and dopamine agonists (the patient declined treatment with levodopa), the patient showed a dramatic improvement with subthalamic nucleus stimulator (STN) implantation. There are no reported cases of use of STN stimulator in HPHA. Conclusion: Hemiparkinsonism-hemiatrophy is a distinct entity that may be clinically and pathogenetically different from idiopathic Parkinson's disease; hence, HPHA needs to be considered as a possible syndrome in patients that have persistent unilateral parkinsonism. As medications are reported to be unhelpful in HPHA cases, early surgical intervention may be an option, such as in our case.
\end{abstract}

RÉSUMÉ: Hémiparkinsonisme-syndrome hémiatrophique somatique. Objectif: Cet article met en lumière le syndrome hémiparkinsonisme-hémiatrophie (HPHA) comme présentation particulière de l'état parkinsonien. Les aspects diagnostiques et thérapeutiques pertinents à la clinique sont revus. Méthode: Nous rapportons un cas de HPHA chez une femme âgée de 21 ans, en bonne santé par ailleurs, et présentons les observations cliniques et radiologiques pertinentes. Nous avons revu la littérature sur les mécanismes cliniques, radiologiques et physiopathologiques de l'HPHA. Résultats: Chez notre patiente, malgré l'absence de réponse aux anticholinergiques et aux agonistes dopaminergiques (la patiente a refusé de prendre de la lévodopa), une amélioration marquée a été observée suite à l'implantation d'un stimulateur dans le noyau sous-thalamique (NST). Il n'existe pas de cas rapporté de l'utilisation d'un stimulateur du NST dans l'HPHA. Conclusion: L'hémiparkinsonismehémiatrophie est une entité distincte qui est peut-être différente de la maladie de Parkinson idiopathique au point de vue clinique et anatomopathologique. L'HPHAdoit donc être considéré dans le diagnostic différentiel des patients qui présentent un parkinsonisme unilatéral persistant. Comme, selon la littérature, la médication n'est pas efficace chez les cas de HPHA, une chirurgie précoce peut être une option valable comme ce fut le cas chez notre patiente.

Can. J. Neurol. Sci. 2002; 29: 184-187

Idiopathic Parkinson's disease (IPD), the most common cause of parkinsonism, is a distinct clinical and pathological entity which results from decreased dopaminergic input into an intact striatum, secondary to degeneration of pigmented neurons in the substantia nigra. In 1981, Klawans ${ }^{1}$ described a new syndrome, hemiparkinsonism-hemiatrophy (HPHA), consisting of hemiparkinsonism and ipsilateral somatic hemiatrophy. Cardinal symptoms included early age of onset $(<40$ years), ipsilateral dystonia, ipsilateral corticospinal dysfunction, slow progression and poor response to levodopa. Three subsequent retrospective case series ${ }^{2-4}$ and numerous single case reports ${ }^{3,5-10}$ have been inconsistent in their use of diagnostic criteria for hemiatrophy. Cases with unquantified somatic hemiatrophy with or without radiological brain hemiatrophy have been variably included, making diagnosis difficult.

We describe a patient with clinically typical HPHA, quantified somatic hemiatrophy, normal magnetic resonance imaging (MRI) and abnormal functional positron emission tomography (PET) scans. Hemiparkinsonism-hemiatrophy is a distinct syndrome with hemiparkinsonism and somatic hemiatrophy. It may result from functional impairment in the nigrostriatal system. Despite this system level similarity to IPD, HPHA is primarily related to dysfunction in the striatum while IPD is primarily related to nigral pathology. Our patient had a

\footnotetext{
From the Department of Clinical Neurological Sciences, London Health Sciences Centre, London, ON Canada

ReCeIVEd MAY 24, 2001. ACCEPTEDin FinAL FORM JANUARY 7, 2002.

Reprint requests to: Mandar S. Jog, Movement Disorders Program, London Health Sciences Centre, 339 Windermere Road, London, ON Canada N6A5A5
} 
dramatic response to subthalamic nucleus stimulation (STN) despite a poor response to anticholinergic and dopamine agonist treatment.

\section{CASE RePORT}

A 21-year-old right-handed woman presented to the movement disorders clinic with a two-year history of gradually worsening rightsided rest tremor, bradykinesia, and foot dragging. Worsening symptoms including small handwriting resulted in her withdrawal from training courses.

She was born full-term with no perinatal complications, but was mildly delayed in the development of head control, speech and certain physical activities. Academic assistance was required throughout the patient's schooling. From an early age, the patient had been aware that her right hand and foot were smaller compared to the other side (e.g. buying shoes).

Medical history included obesity, asthma, and secondary amenorrhea. There was no history of trauma, infection or any other systemic symptoms. There was no significant family history.

On examination, there was visible facial and limb asymmetry with the right side being smaller (Table 1). General examination was normal. Cranial nerve examination was normal apart from hypometric saccades. A large amplitude, distal, 4-6 Hz rest tremor without a postural or action component, rigidity and bradykinesia were present in the right arm and leg. Dystonic posturing of the right hand was observed during repetitive movements on the left. There was a right pronator drift with an extensor plantar response. There was no apraxia, dysmetria or stimulus sensitive myoclonus. Motor examination of the left side was entirely normal. Specifically, there was no tremor, rigidity or bradykinesia. Gait inspection revealed circumduction of the right hip, obvious dystonic posturing of the right foot, and decreased right arm swing. Power, reflexes and sensation were normal and there was no postural instability. The preoperative unified Parkinson's disease rating scales (UPDRS) motor score was 16

Routine biochemistry including liver function tests, lactic acid, serum and 24-hour urinary copper, ceruloplasmin, ESR and vasculitis screen were normal

An MRI of the head was normal. Fluorodopa (FDP) PET scanning showed a left-sided reduction in striatal FDP uptake involving both the caudate and the putamen equally. Fluorodeoxyglucose (FDG) PET scanning showed a decrease in glucose metabolism in the left striatum.

Over the next nine months the patient was unsuccessfully tried on 60 $\mathrm{mg}$ of trihexyphenidyl with $40 \mathrm{mg}$ of bromocriptine. Treatment with levodopa was recommended, but the patient and family refused further trials of medications. The risks and benefits of neurosurgical intervention were then reviewed and the patient elected to proceed directly to surgery. All medications were then discontinued and never restarted.

The left subthalamic nucleus was targeted using standard stereotactic localization protocol including Leksell frame application and a volumetric T1-weighted MRI scan. Apreliminary target was chosen $4 \mathrm{~mm}$ below and $12 \mathrm{~mm}$ lateral to the anterior commissure-posterior commissure (AC-PC) line and $1 \mathrm{~mm}$ posterior to mid AC-PC line. The left subthalamic nucleus was explored through a coronal parasagittal twist drill opening using microelectrode recording and stimulation. Standard data acquisition equipment was used to observe and listen to the neuronal responses. Left subthalamic localization was confirmed in two ways. Firstly, intraoperatively, boundaries of the thalamus and the
Table 1: Quantification of somatic hemiatrophy in a righthanded HPHApatient.

Measurements were made using published landmarks. ${ }^{18}$

\begin{tabular}{lrcc}
\hline Measurement (cm) & Left & Right & Difference \\
palpebral fissure length & 3.6 & 3.2 & 0.4 \\
upper limb length & 69.7 & 68.5 & 1.2 \\
total hand length & 17.4 & 16.8 & 0.6 \\
lower limb length & 77.0 & 75.4 & 1.6 \\
foot length & 22.0 & 21.0 & 1.0 \\
\hline
\end{tabular}

Table 2: Summary of currently existing case series on HPHA. Not all studies provided information on the listed sub-headings.

\begin{tabular}{lccc}
\hline & Buchman $^{2}$ & Giladi $^{3}$ & Przedborski \\
Number of patients & 15 & 11 & 6 \\
Mean age of onset (yrs) & $43.7(31-61)$ & $38.1(18-54)$ & $41(23-59)$ \\
Ipsilateral dystonia & $67 \%$ & $27 \%$ & $83 \%$ \\
Ipsilateral UMN signs & $53 \%$ & $9 \%$ & $17 \%$ \\
Response to treatment & $78 \%$ & $70 \%$ & $33 \%$ \\
Time to treatment (yrs) & 14.2 & not reported & not reported \\
Abnormal birth history & $50 \%$ & $0 \%$ & $0 \%$ \\
Abnormal MRI or & & & $67 \%$ \\
CTneuroimaging & $8 \%$ & $55 \%$ &
\end{tabular}

$\mathrm{UMN}=$ upper motor neuron; CT = computed tomography

quiet physiological prelemniscal radiations were defined. The subthalamic nucleus was then immediately below this area. Once the electrode was advanced to the stereotactic area of the subthalamic nucleus, response of units demonstrating spontaneous firing synchronous with the patient's tremor as well as units responding to movement of the contralateral limbs were also observed. Microstimulation in this region produced tremor arrest. Spike morphology and spike train analysis was not performed. Secondly, the final location of the stimulating electrode was subsequently confirmed by a postoperative MRI scan.

A quadripolar deep brain stimulating electrode (model 3389, Medtronic Inc., Minneapolis) was inserted through a burr hole into the left subthalamic nucleus. In addition to standard stereotaxy, microelectrode recordings were used to guide target location. Two days later, under general anesthetic, the deep brain electrode was attached to an implanted neurostimulator placed subcutaneously in the infraclavicular region (Itrel 2, Medtronic Inc., Minneapolis). The left subthalamic nucleus was stimulated using monopolar stimulation (frequency $-185 \mathrm{~Hz}$; pulse width $-90 \mu \mathrm{sec}$; amplitude -2.1 volts) resulting in complete cessation of arm and leg tremor, reduction of rigidity and dystonia.

There was sustained improvement of tremor, bradykinesia, handwriting and foot dystonia at nine month follow-up on the left side. The patient was on no medication. The UPDRS motor score on the 
affected side with stimulator "on" was 4 (off stimulation motor score 16). Although formal quality of life measures were not performed, the patient was able to return to her studies.

\section{Discussion}

This paper reviews the spectrum of clinical and neuroradiological features and possible pathogenetic mechanisms that have been used to define HPHA. Case series have variably included patients with somatic and radiological hemiatrophy, either singly or in combination (Table 2). $\cdot^{2-4}$ In addition, quantification of hemiatrophy has been inconsistently reported and has included visual, radiological (hands and feet $\mathrm{x}$ rays) and simple hemi-body measurements. , $^{5,8,11}$ This variation in patient inclusion criteria described in the HPHAliterature will be discussed.

\section{Clinical characteristics}

Klawans ${ }^{1}$ defined the clinical features of HPHAin four cases, including early age of onset, ipsilateral dystonia and upper motor neuron findings, slowly progressive course, poor response to levodopa and possible association with perinatal injury. Clinical features from three subsequent case series are summarized in Table 2 .

Hemiparkinsonism-hemiatrophy is reported to have an earlier age of onset than IPD. One retrospective case series $^{2}$ with 15 HPHAand 31 IPD patients had mean ages of onset of 43.7 years and 55 years respectively. Two subsequent studies found mean ages of onset of HPHA to be 38.1 and 41.0 years. ${ }^{3,4}$

Other signs such as pretreatment dystonia and upper motor neurons signs occur rarely in early IPD, reported in $2.5 \%$ of patients with IPD ${ }^{12}$ and $27-83 \%$ of patients with HPHA. $3,4,13$ Ipsilateral upper motor neuron signs were less common and more variable in patients with HPHAwith a 9-53\% occurrence. ${ }^{2-4}$

Despite case reports of a lack of response of HPHA to levodopa, only three case series have reported treatment response in a small patient cohort. ${ }^{3}$ Treatment response to levodopa was variably seen in $33-78 \%$ of patients. ${ }^{3,4}$ Unfortunately patient inclusion criteria were variable (Table 2) making conclusions difficult.

Hemiparkinsonism-hemiatrophy appears to progress at a significant slower rate than mild IPD (Hoehn and Yahr I and II), with a mean duration to levodopa initiation of 14.2 years (HPHA) versus 3.7 years (IPD) $(\mathrm{p}<.001){ }^{2}$

An abnormal birth history (prolonged labor or delivery, traumatic delivery, breech presentation, premature delivery, or neonatal hypoxia) has been reported in 50\% HPHA patients in one case series. ${ }^{2}$ In contrast to this, two subsequent studies ${ }^{3}$ found no history of perinatal complications in HPHA patients.

Idiopathic and structurally induced hemiparkinsonism and hemiatrophy can occur in other clinical conditions. Predominantly unilateral parkinsonism can be seen in early cortical-basal ganglionic degeneration and IPD. ${ }^{14}$ Structurally induced somatic hemiatrophy with subsequent development of a clinical syndrome similar to HPHA has been reported with nigral, ${ }^{5}$ thalamic and rostral brain stem lesions. ${ }^{6}$ In addition, ipsilateral hemiatrophy has also been reported in patients with asymmetric dopa-responsive dystonia. ${ }^{15}$ Dopa-responsive dystonia is thought to be linked to an inherited loss of an enzyme (GTP cyclohydrolase) which is required for dopamine production. Unlike HPHA, these patients respond to levodopa administration.

\section{Neuroimaging}

Hemiparkinsonism-hemiatrophy was originally described as a clinical syndrome without structural radiographic abnormalities. ${ }^{1}$ Subsequent case series that have retrospectively reviewed available neuroimaging (CT and MRI) have found a variable frequency of occurrence of radiological abnormalities ranging from $8-67 \% .^{2,4,5}$ These changes have included enlarged lateral ventricles, cortical, sub-cortical or basal ganglia atrophy (Table 2).

Although not part of the original description ${ }^{1}$ of HPHA, metabolic functional abnormalities have been consistently observed in HPHA. Despite inclusion of patients with and without structural neuroimaging abnormalities, functional imaging has shown metabolic dysfunction in all the patients with somatic hemiatrophy. 4,16

$\left[{ }^{18} \mathrm{~F}\right]$-fluorodopa PET examines the function of the nigrostriatal dopamine pathway. Fluorodopa PET scanning has been studied in HPHA, IPD and normal subjects. Studies show a reduction in contralateral striatal FDP uptake in the HPHA patients when compared to normal controls (Table 2). ${ }^{5}$ Such reduction in the striatal fluorodopa uptake was also reported in patients with unilateral IPD. ${ }^{5}$ A single HPHA patient showed bilateral reduction in FDP uptake despite unilateral symptoms/signs. ${ }^{4}$ Despite this, HPHA patients showed a minimal response to levodopa. ${ }^{4}$

$\left[{ }^{18} \mathrm{~F}\right]$-fluoroethylspiperone (FESP)-PET scanning assesses striatal dopamine D2 receptor binding capacity. Fluoroethylspiperone is a ligand, which binds in vivo to high affinity dopamine D2 receptors. In patients with a poor response to levodopa, one might have predicted a reduction in striatal D2 receptor binding capacity. In two HPHA patients with a poor response to levodopa, FESP scans did not reveal a reduction in FESP binding. ${ }^{7,10}$ In a single study of $\left[{ }^{123} I\right]-2-\beta$-carbo-methoxy-3- $\beta-(4-$ iodophenyl)-tropane (ICIT) single photon emission computed tomography scanning evaluating dopamine transporter activity in a patient with HPHA, there was a marked bilateral but asymmetric reduction in striatal tracer uptake?

$\left[{ }^{18} \mathrm{~F}\right]$-fluorodeoxyglucose PET scanning has also been studied in these groups. Utilising differences in regional glucose metabolism, FDG PETexamines the function of the striatum. ${ }^{4,16}$ A reduction in glucose metabolism was seen in the contralateral basal ganglia in all HPHA subjects. In contrast, patients with unilateral IPD and normal controls did not show any significant abnormality. Human FDG PET studies and animal models of unilateral IPD have consistently shown normal or increased contralateral metabolism in the caudate and lentiform nuclei. ${ }^{10,16,17}$

\section{Pathogenesis}

Klawans ${ }^{1}$ postulated that the lack of levodopa response in HPHA was related (at least in part) to primary striatal dysfunction resulting from a perinatal insult.

Positron emission tomography scanning results discussed above may shed light on the pathogenetic mechanisms of HPHA. ${ }^{4,10,16,17}$ The contralateral FDP uptake was significantly decreased in both HPHA patients and unilateral IPD patients. ${ }^{4}$ This abnormality suggests that both conditions have dysfunction 
along the nigrostriatal pathway but does not separate them. However, the reduction of glucose uptake in the striatum in HPHA suggests that primary loss /dysfunction is at the level of the striatal neurons themselves and may explain the lack of a consistent response to levodopa. ${ }^{4}$ In contrast, the preservation of glucose uptake in the striatum in IPD and response to levodopa is expected, since the pathology of this condition is primarily nigral.

$\left[{ }^{18} \mathrm{~F}\right]$-fluoroethylspiperone-PET and ICIT SPECT scanning results showing normal dopamine D2 receptor binding but abnormal dopamine transporter activity suggest a nonreceptor mechanism for the striatal dysfunction in HPHA.

To date, there has been no postmortem examination of an HPHA brain. Histopathology and biochemical tests of HPHA brain tissue will be necessary to understand its pathogenesis. Nevertheless, studies outlined above do favour a primary, striatal etiology to the HPHA syndrome.

Our case is clinically typical of HPHAwith early age of onset, ipsilateral dystonia, upper motor neuron signs, and a slowly progressive course. Although our patient was not tried on levodopa, respecting her wishes, she did demonstrate a complete lack of response to anticholinergic and dopamine agonist treatment. In addition, the abnormal FDG and FDP PET studies with normal MR imaging were consistent with the literature and would therefore favour primary striatal pathology.

\section{CONClusion}

Hemiparkinsonism-somatic hemiatrophy syndrome was first described in 1981 by Klawans ${ }^{1}$ as a form of secondary parkinsonism. Hemiparkinsonism in HPHA occurs after a lag period and ipsilateral to the side of somatic hemiatrophy. The somatic hemiatrophy is congenital, visibly obvious, and of variable involvement (e.g. face, limbs, trunk). With only one exception, ${ }^{8}$ reports of HPHAhave not included formal measures of somatic hemiatrophy.

Our case and other retrospective case analyses suggest that HPHA may differ from IPD in several important respects. First, the response of HPHA patients to traditional antiparkinsonian medications appears to be quite variable. Secondly, the longterm prognosis in HPHAis probably better than that in unilateral IPD due to slower disease progression. Thirdly, the cellular pathology of IPD is nigral, as compared to the postulated striatal dysfunction in HPHA. Finally, success of deep brain stimulation in IPD requires response to standard dopaminergic replacement therapy. Our patient had a dramatic response to STN stimulation in the absence of any improvement with anticholinergic and dopamine agonist therapy.
Hemiparkinsonism-somatic hemiatrophy is therefore an interesting model of a unique form of parkinsonism.

\section{REFERENCES}

1. Klawans HL. Hemiparkinsonism as a late complication of hemiatrophy: a new syndrome. Neurology 1981;31:625-628.

2. Buchman AS, Goetz CG, Klawans HL. Hemiparkinsonism with hemiatrophy. Neurology 1988;38:527-530.

3. Giladi N, Burke RE, Kostic V, et al. Hemiparkinsonismhemiatrophy syndrome: clinical and neuroradiologic features. Neurology 1990;40:1731-1734.

4. Przedborski S, Giladi N, Takikawa S, et al. Metabolic topography of the hemiparkinsonism-hemiatrophy syndrome. Neurology 1994;44:1622-1628.

5. Lang AE. Hemiatrophy, juvenile-onset exertional alternating leg paresis, hypotonia, and hemidystonia and adult-onset hemiparkinsonism: the spectrum of hemiparkinsonismhemiatrophy syndrome. Mov Disord 1995;10:489-495.

6. Leenders KL, Frackowiak RS, Quinn N, et al. Ipsilateral blepharospasm and contralateral hemidystonia and parkinsonism in a patient with a unilateral rostral brainstem-thalamic lesion: structural and functional abnormalities studied with CT, MRI, and PETscanning. Mov Disord 1986;1:51-58.

7. Marchioni E, Soragna D, Versino M, et al. Hemiparkinsonismhemiatrophy with brain hemihypoplasia. Mov Disord 1999;14:359-364.

8. Martinelli P, Scaglione C, Capocasa M, Dalpozzo F, Contin M. Hemiparkinsonism-hemiatrophy: a new observation. J Neurol 1998;245:180-182.

9. Costa B, Zanette G, Bertolasi L, Fiaschi A. Hemiparkinsonismhemiatrophy syndrome: neuroradiological and neurophysiological findings. Eur Neurol 1994;34:107-109.

10. Przedborski S, Goldman S, Levivier M, et al. Brain glucose metabolism and dopamine $\mathrm{D} 2$ receptor analysis in a patient with hemiparkinsonism-hemiatrophy syndrome. Mov Disord 1993;8:391-395.

11. Halperin G. Normal asymmetry and unilateral hypertrophy. Arch Intern Med 1931;48:676-684.

12. Kidron D, Melamed E. Forms of dystonia in patients with Parkinson's disease. Neurology 1987;37:1009-1011.

13. Buchman AS, Comella CL, Leurgans S, Stebbins GT, Goetz CG. The effect of changes in head posture on the patterns of muscle activity in cervical dystonia (CD). Mov Disord 1998;13:490-496.

14. Giladi N, Fahn S. Hemiparkinsonism-hemiatrophy syndrome may mimic early-stage cortical-basal ganglionic degeneration. Mov Disord 1992;7:384-385

15. Greene PE, Bressman SB, Ford B, Hyland K. Parkinsonism, dystonia, and hemiatrophy. Mov Disord 2000;15:537-541.

16. Przedborski S, Goldman S, Giladi N, et al. Positron emission tomography in hemiparkinsonism-hemiatrophy syndrome. Adv Neurol 1993;60:501-505.

17. Eidelberg D. Positron emission tomography studies in parkinsonism. Neurol Clin 1992;10:421-433.

18. Hall JG, Froster-Iskenius UG, Allanson JE. Handbook of Normal Physical Measurements. New York:Oxford University Press 1989. 